\title{
Sustainable Health Equity
}

\author{
Mary Kerich \\ University of Maryland Medical Center Baltimore, MD \\ USA \\ E-mail: Marykerich1@gmail.com
}

$\begin{array}{lrr}\text { Received: May 26, } 2018 \quad \text { Accepted: July 13,, } 2018 \quad \text { Published: July 13, } 2018 \\ \text { doi:10.5296/jmr.v10i3.13323 } & \text { URL: https://doi.org/10.5296/jmr.v10i3.13323 }\end{array}$

\begin{abstract}
Sustainable Health Equity (SHE) is a progressive national initiative that promotes the health of individual citizens and communities by modifying socio economic and environmental factors that correspond to social determinants of health. The sustainable health equity focuses on modalities to engage policy actors, stakeholders and decision makers to conceptualize an actionable public health policy. The collaborative national initiative is multifaceted which is principally to provide consistent health care that does not vary according to demographics like gender, age, ethnicity, socioeconomic and geographical location. Cultural congruent and universal health care are the pillars to health equity in the United States. The Stakeholders include government, researchers, civil societies, health care professionals, providers and the public. An actionable SHE policy will advance public confidence in the executive, judiciary, legislators and public officials. Multidisciplinary and multilevel engagement is essential in addressing health disparity in the United States. Strategies to foster political power, create awareness, advocate for high-quality health care progress evidence- based practices, research and equal allocation of material and resources. SHE prospective is inherent with the secretary of the Department of Health and Human Services unequivocal resonance in public service, and exemplary leadership.
\end{abstract}

Keywords: SHE, public policy, health disparities, socio economics, discrimination, social inequity 


\section{Introduction}

The Institute of medicine defines racial/ethnic disparities as differences in health care services received by two groups that are not due to differences in the underlying health care needs or preferences of members of the groups (Cook, McGuire, \& Zaslavsky, 2012). Disparities occur due to the operation of health care systems, legal, political discrimination of underprivileged populations. Health disparity is well documented, and literature demonstrates the link between underprivileged socioeconomic and ethnic minority groups with reduced health care access and poor health outcomes (Srinivasan \& Williams, 2014).Healthy People 2020 define health disparity as a difference in health that is closely linked to social, economic, and environmental disadvantages (Tunkel, 2014). The center for disease control define health inequities as types of unfair health difference closely linked with social economic or environmental disadvantages that adversely affect different demographics. Empirical evidence demonstrates correlation between deprived socioeconomic status and poor health that raise concerns over the income inequality in the United States. Social determinants of health include early life, education, employment / working conditions, food security, health services and housing.

The United States spends more than double per capita on health care in comparison with other developed countries, a phenomenon that importune public opinion (Esteban, \& Dickinson, 2013).Congress has the budgetary decision- making authority that control revenue and spending levels, by approving or obstructing appropriations bills. The legislative branch reviews the presidential budget proposal, establish priorities and negotiate budget resolutions before sending the appropriation bill to the president for approval (Anderson, 2015). Advocacy for SHE health is advanced by creating awareness about the impact of health disparities on chronic diseases and poor health outcomes and the high cost of treatments. Type 2 diabetes is a typical example of chronic disease that predisposes individuals to cardiovascular and kidney diseases. Uncontrolled diabetes leads to kidney failure and require expensive treatments like renal dialysis and kidney transplant.

\section{Conceptual framework}

The theory of attribution provides a conceptual framework for framing the questions that contour appropriate responses from the decision makers and the public, by drawing on personal and societal responsibilities in health equity (Lundell, Niederdeppe, \& Clarke, 2013).SHE advances preventive attributes of health like healthy eating habits to prevent obesity, and smoking cessation, support initiative that address social issues like unemployment and poverty. Health comparative questions based on specific demographics will advance the discourse for societal responsibility in addressing health disparities. The objective is to revolutionize the health care industry by advancing the social and health equity agenda. Deliberate effort is required from the government and non-governmental agencies to modify class and racial health disparities and eliminate discrimination, biases and stereotype in the public domain.

Public policy actors must address individual and societal responsibilities concurrently because both aspects are intertwined. The irrefutable danger of health care disparity and the 
ensuing public health inference enroll social justice and the need for future focused research on health equity and social determinants to achieve highest level of health possible for all people (Srinivasan \& Williams, 2014). A holistic approach to health policy requires collaboration among policy actors, public awareness, and education on individual and societal responsibilities that promote the continuum of health and social responsibility.

Social determinants of health require integration and inclusive approach that breaks down interdisciplinary and jurisdiction barriers with the objective to support SHE public policy. Innovative strategies to effectively manage the social determinants of health and eliminate unfavorable environmental factors that contribute to poor health are the essence of social justice. Targeted and responsive education will build a capacity for positive social change and advocacy for human rights and health equity. Anderson (2015) expound on the role of nongovernmental participants in public policy such as interest groups, political parties, research organization, public media and the public. SHE is a collaborative venture that links the deliberate efforts of health policy practitioners, community members, health care providers, government officials, and private sector to improve public health and the quality of life for the poor and underserved populations (Eltom, Tchounwou, \& Rice, 2011).

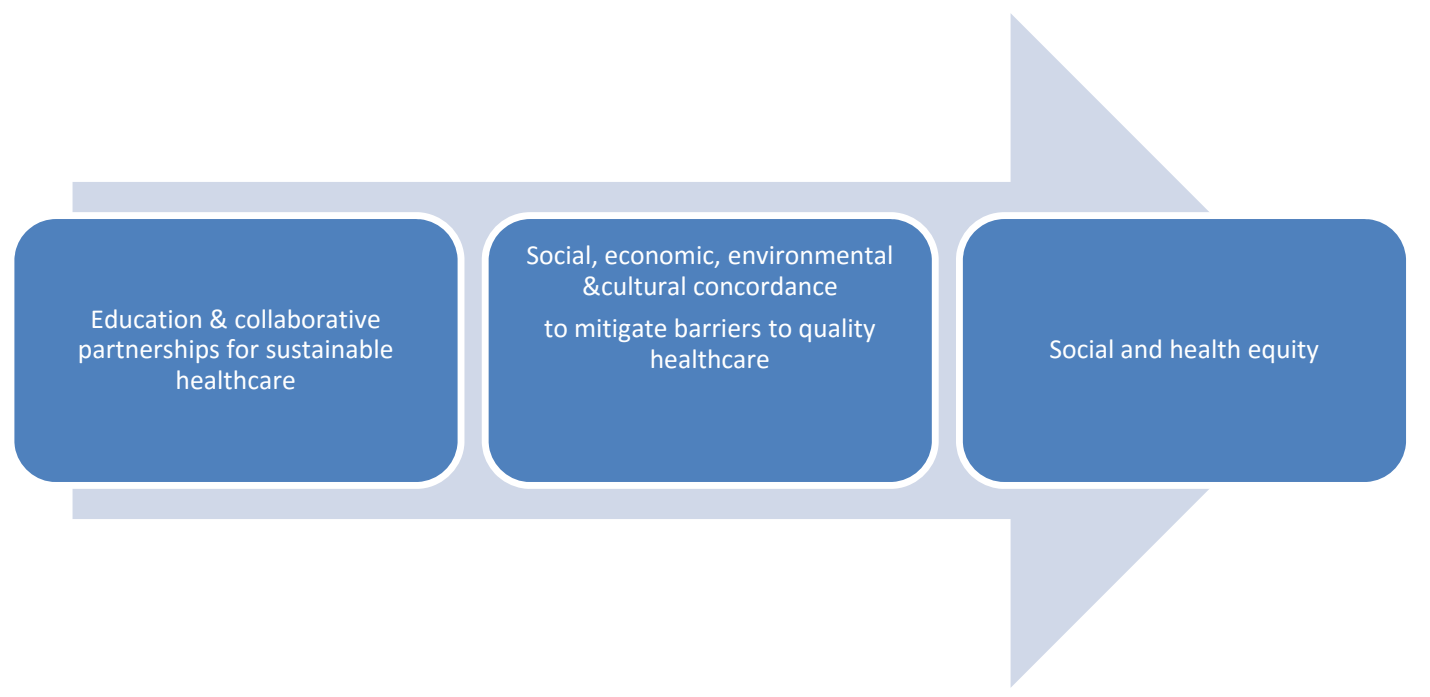

Figure 1. Social and health equity roadmap

The road map towards social and health equity anchor on the modification of societal determinants of health and practical strategies to develop and maintain a culture where ethical behavior, human integrity and resources are aligned. The social and health disparity gap in the United States require innovative strategies that utilize knowledge and empirical data to modify the social determinants of health for best health outcomes (Eltom, Tchounwou, \& Rice, 2011).Socioeconomic and environmental factors are associated with health disparities and poor health outcomes, and correlate with the discrepancy in the allocation of material and resources among marginalized communities (Northridge, \&Freeman, 2011). 


\section{Issue Analysis}

Health disparity is a public health problem in the United States and is evident in the health screening, treatments and health outcomes (Fox \& Scanlan, 2014).Actionable social and health equity policy require understanding the public perception of individual behaviors and personal responsibility associated with health disparities (Lundell, Niederdeppe, \& Clarke,2013). Policymakers must understand the social, cultural and environmental factors that contribute to health disparities as a foundation for innovative strategies that promote social and health equity in the United States. Notably, the disproportional prevalence of HIV/AIDS and poor health outcomes among the minority populations correlate with race, neighborhood, and socio-economic status (Fox \& Scanlan,2014). Researchers demonstrate that questionable prescriptions, higher copayments for health screening, and gaps in the quality of health care is rampant in the Hispanic and African American compared to the white communities (Fox \& Scanlan, 2014).Public awareness of health disparities and the proposed SHE policy will enhance public support that pressures the political battery to a favorable action. Universal health care increase access while cultural congruent care promotes individualized care that promote healthy lifestyles.

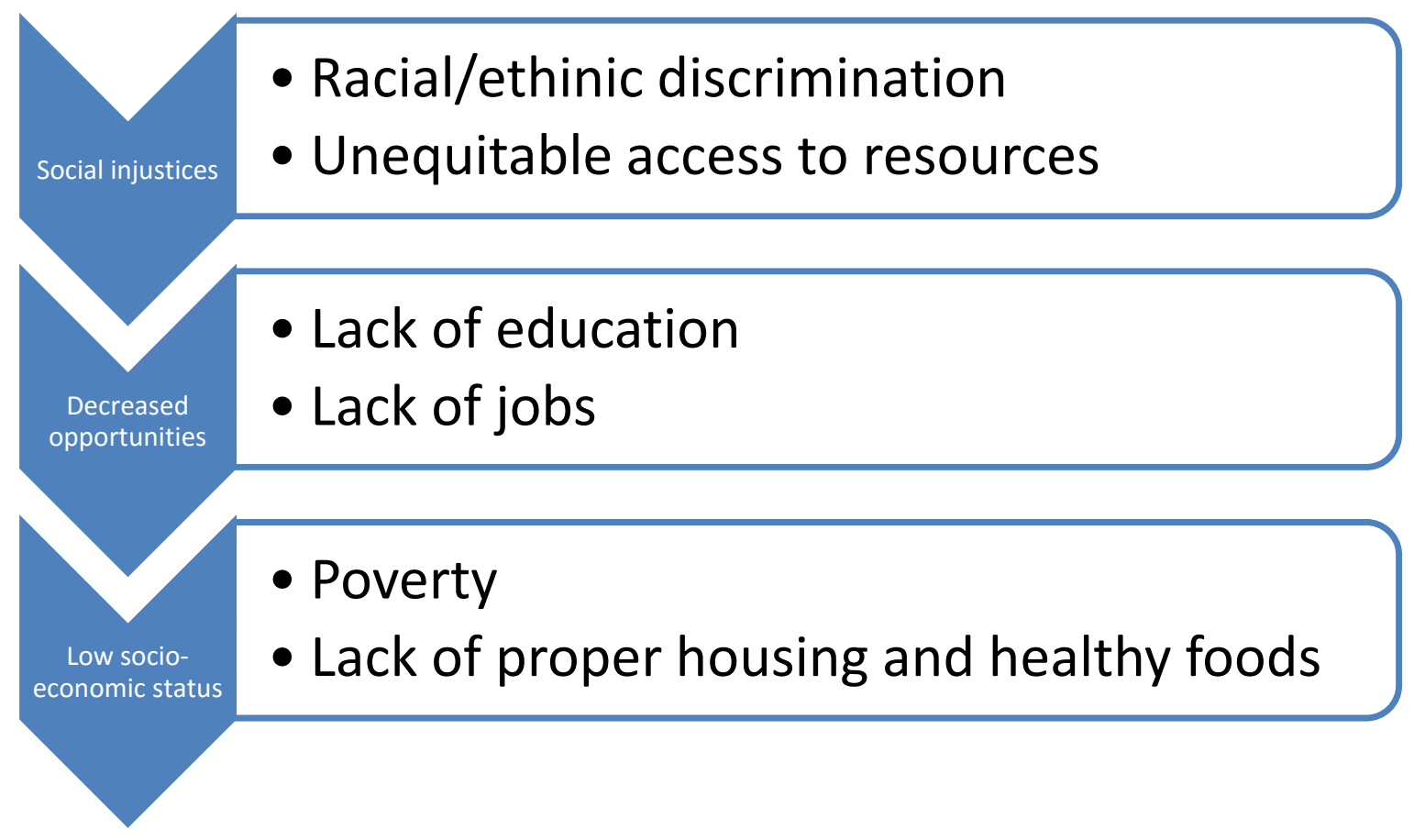

Figure 2. Societal Determinants of Health Disparities

Social injustices like discrimination and unequal distribution of resources and material decrease opportunities in education and employment, and increase poverty, lack of safe housing and healthy foods. Ethnicity as a social determinant of health includes the individual behaviors, social status, education, environmental pollution, social support, neighborhood and housing quality (Lundell, Niederdeppe, \& Clarke, 2013). Health care and healthy life styles have been the main focus of health promotions by clinicians and public health officers. The 
perception that an individual is responsible for their health is widely accepted, although providing an enabling environment is equally important. Initiatives like employee health, healthy eating habits, regular exercises and avoidance of drug and alcohol abuse promote health outcomes.

Leverage of the policymakers, health practitioners and news media advances the social responsibility agenda. African Americans and Hispanic communities encounter socioeconomic challenges like lack of higher education, unemployment, discrimination and poverty. Consequently, Hispanics, African-Americans, and Native American individuals suffer disproportionately from higher rates of hypertension, sexually transmitted diseases and different forms of cancers (Rivera, 2014).

\section{Racial/Ethnicity}

Racial/ethnicity is attributed to the disproportionate prevalence of chronic diseases like cardiovascular, diabetes and HIV/AIDS among African American in the United States.

In 2012, blacks accounted for $47 \%$ of persons who received a human immunodeficiency virus (HIV) diagnosis, and in 2011, they accounted for $43 \%$ of persons living with HIV. During 2008-2011 more deaths among black persons living with HIV occurred each year than among any other race/ethnicity (Siddiqi, Hu,\& Hall, 2015).Subsequently, in 2012, an estimated 8,165 (48\%) deaths occurred among African Americans living with HIV, which is 1.5 times the number of deaths among whites $(5,426)$ and 3.2 times the deaths among Hispanics or Latinos $(2,586)$ as noted by (Siddiqi, Hu, \& Hall, 2015).Researchers demonstrate that Racial/ethnic disparities vary among states and require innovative strategies to increase health care utility by African Americans living with a diagnosis of HIV infection. Medical treatment plans and health education should be tailored to meet the specific needs of the population.

\section{Homelessness}

The phenomenon of homelessness is directly linked to poor health outcomes and affects about $1 \%$ of the United States population annually a factor that deters access and adherence to health care in a demographic that disproportionately suffer high rates of chronic and acute diseases (Rabiner, \& Weiner, 2012). Families with children are one of the fastest growing populations of homeless, consisting of $41 \%$ of the homeless population (Rabiner, \& Weiner, 2012). Diverse factors that contribute to homelessness include poverty, inequity in the distribution of resources, and mental illness. Subsequently, Lack of health insurance, frequency in changing geographical locations, food insufficiency and exposure to contaminants increase emergency room visits, hospitalization and poor health outcomes among the homeless. Delay in receiving appropriate Medical care, poor nutrition and violence increase the morbidity and mortality rate amongst the homeless. Providing stable housing, cultural congruent health education and means of communication will enhance adherence to treatment and follow up plans for the homeless. 


\section{Discrimination}

Analysis of the social determinant of health receives limited attention, despite the empirical evidence of health disparities and the associated social economic factors. Unequal treatment by those in authority compels the notion of discrimination and the bureaucracy of allocating material and resources is elusive in the prevalence of political polarization. Functions of the administrative government are reflective of the interface between politics, executive, congress, judiciary and interest groups. Underserved population has less trust in the administrative government due to perceived discrimination in health care, education, and allocation of resources and materials.

Health disparities draws the attention of interest groups due to the causative factors like poverty, inadequate health care, less education, poor housing and lack of access to healthy foods (Rivera, 2014). Genetic and biomedical factors interface with social and environmental conditions to cause disease and disability; exclusion of disadvantaged populations from the clinical trials curtails appropriate medical treatments and evidenced based practice. Scholars and researchers should examine the social determinants of health and disseminate the information with an objective to create public awareness and inform the policymakers.

\section{Proposed Solutions}

The frameworks to realize SHE is founded on the principles that modify social determinants of health and environmental factors that contribute to poor health. Strategic planning that progress social justice, social inclusion, equal opportunities, and access to affordable and safe housing, education and health care form the building blocks toward social and health equity in the United States. 


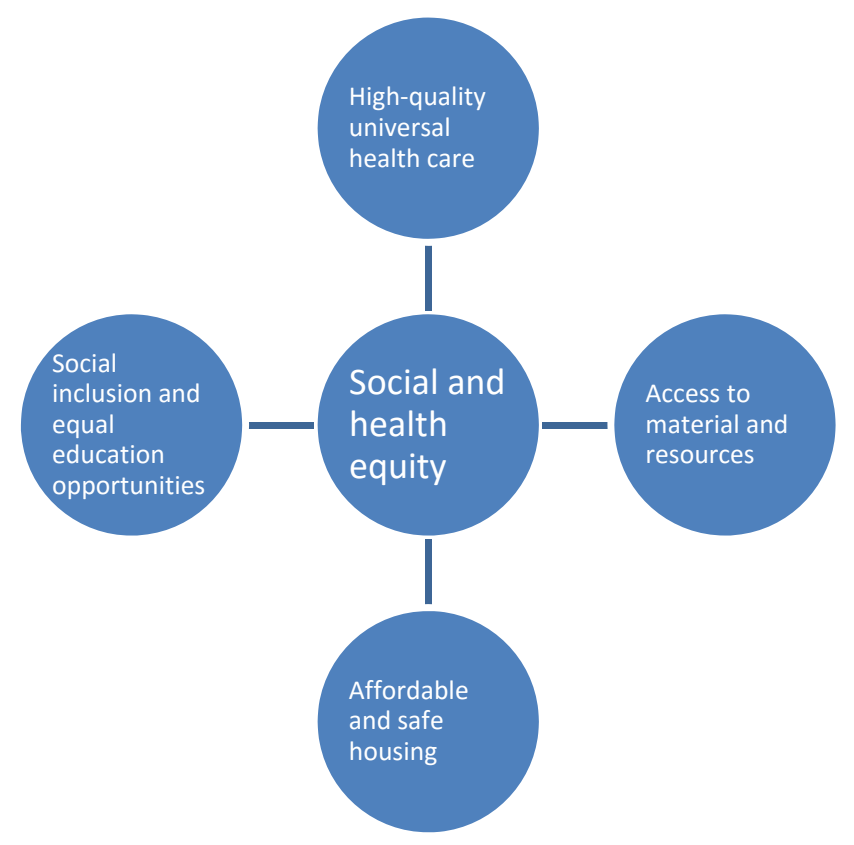

Figure 3. Visual presentation of areas of propose solutions

\section{Personal and Societal Responsibilities}

Personal and societal responsibilities align to promote the health of individuals and communities. Societal responsibility includes evolution of the health care system to increase access for the disadvantaged populations, and equal distribution of resources affords opportunities for adequate education and employment that promote socio-economic status. Health care professionals have the obligation to provide high-quality, cultural congruent health care and education to all persons irrespective of race, creed, sex, age or any other demographic attributes. The primary responsibility of health primarily lays within the individual scope of making healthy choices, and effectively interacting with significant others, families, community of faith to build a supportive social network that progress personal wellbeing. Public awareness, impact of individual and societal responsibilities will promote the continuum of health and social responsibility. However, the public must have confident in the administrative government for a meaningful participation in the policy process that incorporate the translation of historic and cultural factors to real life experiences and the degrees of responsiveness by the Government (Miller,2011). 


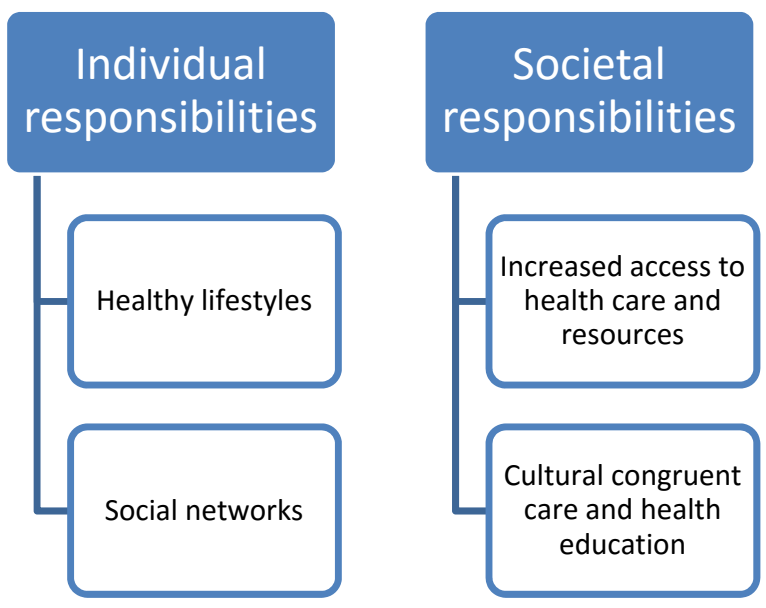

\section{Equal Allocation of Material and Resources}

Figure 4. Personal and societal responsibilities in health equity

Social determinants of health require integration and inclusive approach that breaks down interdisciplinary and jurisdiction barriers with the objective to support SHE public policy. Innovative strategies to effectively manage the social determinants of health and eliminate unfavorable environmental factors that contribute to poor health are the essence of social justice. Targeted and responsive education will build a capacity for positive social change and advocacy for human rights and health equity. Anderson (2015) expound on the role of nongovernmental participants in public policy such as interest groups, political parties, research organization, public media and the public.

Access to resources and material is a conduit to increase supply for health foods and affordable housing. Government incentives and subsides are initiatives designed to improve the living conditions and lives of the underprivileged populations. Equal education opportunities transform lives through capacity building to make informed decisions, secure jobs and promote healthy lifestyles. Community environments are associated with health behaviors like physical activities, preventive care, alcohol, and sexual behaviors and health outcomes like obesity, cardiovascular and mental health (Fox Wetle, \& Scanlan, 2014).

Equitable allocation of resources support community investments and design neighborhoods that minimize behavioral and environmental health hazards like contamination, infection, mental stress and injury. A collaborative effort between government agencies in health, social services, education, environment and housing constitute a closed loop that modifies societal determinants of health. Education and public outreach programs aim to create awareness on individual and community benefits of social economic equity through increased opportunities and political power.

\section{Affordable and Safe Housing}

Affordable and safe housing is achieved through effective urban planning and government subsides. Improved health amities and other environmental factors are achieved through equal distribution of government and community resources. Removal of environmental 
hazards and pollutants such as lead paint, mold, gas emissions and unhealthy foods and alcohol ensure a safe and clean environment that promote health and wellbeing of the residences (Northridge, \&Freeman, 2011). Some ethnic-racial individuals live in poor neighborhoods, Social institutions and communities, and are subjected to stigma that cause emotional stress; catalyst to poor health outcomes (Rivera, 2014).Although homelessness is not a disease process, medical professionals need to be aware of a patient's living status and factor in the social aspect in the treatment plans (Rabiner, \& Weiner, 2012). Improved urban housing, innovative government subsidy for low income population provide an exemplary social services administration that progress social and health equity. The United State department of housing and urban development secretary Julian Castrol plays a vital role to ensue transparency and equal opportunity through Federal Housing Administration public services. The office of housing should fulfill its mission and contribute to the building and preserving healthy neighborhoods and communities.

\section{Universal Health Care}

High-quality health care is achieved through universal health insurance that increases access and expand preventive care that reduces illness and lowers the cost of health care. Institutional change and administrative restructuring of health and social services to create universal health care will increase health care access irrespective of social economic status. Empirical evidence on societal determinants of health will advance best practices that promote culture congruent care. The medical fraternity requires cultural competency education that progress patient-centered medical model, and advocate for the inclusion of minority health care professionals in leadership positions as observed by (Feroe, \&Loebs, 2015). Increasing mental health funding, sponsorship for research studies that focus on health disparities and re-engineering the health care delivery model will advance health equity in the United States.

The hallmark of SHE is a concordance of social economic equity and modification of the social determinants of health that correlate with economic paucity. Healthcare Providers must recognize multiple sources of stereotypes, prejudice and discrimination that trigger and maintain a variety of social psychological processes that increase the risk for detrimental health associated with ethnic-racial groups (Rivera, 2014).Equal access to education, health care, and resources will progress health equity. There is a need to safeguard the future of Patient Protection and Affordable Care Act (PPACA) that increase health care access through Medicaid expansion, and provide equal education through education reform.

SHE focuses on structured community initiatives that positively impact healthy life styles and keep social and health equity on the public agenda. The Adoption of electronic health records forms a foundation for universal health care. Health disparities, rising cost of health care and the ethical considerations resonate with the legislative institutions and the policy actors in the social and health equity. Health Education and other outreach efforts provide public awareness on healthy lifestyles and health education on diseases like stroke, cancer, asthma, diabetes, drug addiction, mental illness, and cardiovascular conditions. Cultural competence 
among health care professionals will advance evidence-based practices that promote best health outcomes.

The establishment of the offices of minority health within government agencies, and the National Partnership for Action increase public outreach and awareness (Takamura, 2014).Advocacy for the right to health care is framed to eliminate discrimination based on personhood, and the socio-economic impact. Social inclusion, affordable housing and education promote social economic and health equity.

\section{Equal Education Opportunities}

The National dialogue on social and health disparities affecting venerable populations must conceptualize investments in the youth as the future generation. Youth empowerment programs are designed to equip young people ages 12 to 18 years with coping skills through mentorship and health education. Capacity building among the young people promotes healthy lifestyles. Public school officials and educators need to restructure curriculums and learning environments to meet the prevailing community needs and challenges.

Standardized testing as prescribed by the United States education policy is contested by educators, parents, and communities. The controversy is mainly over the features of skewed cultural insensitive test content, and ineffective method of computing K-12 workforce and teacher evaluation (Robelen, 2011).Standardized test speaks to the main stream culture as common knowledge but is perceived to be culturally irrelevant in a diverse population. Teachers express dissatisfaction over the amount of time wasted in the testing process, and loss of teaching time. Federal provision of No Child Left behind Act need can be interpreted in the context of equity in education, health care and social economic opportunities that promote social and health equity. Information gap and disconnect between legislators, civil servants, medical professionals, educators and the general public stress the importance for innovative strategies to involve all the internal and external stakeholders in the policy process. 


\section{Policy Recommendations}

Table 1. Summary of social and health equity framework priority strategies

\begin{tabular}{|l|l|}
\hline Priority Strategies & Intended Results \\
\hline 1.Foster political will & $\begin{array}{l}\text { Increase decision makers understanding of } \\
\text { the impacts of health disparities through } \\
\text { dialogue and public media coverage. }\end{array}$ \\
\hline 2. Create awareness and education & $\begin{array}{l}\text { Increase public awareness about the benefits } \\
\text { of social and health equityand promote } \\
\text { cultural competency in learning institutions } \\
\text { and in the health care models. }\end{array}$ \\
\hline $\begin{array}{l}\text { 3. Interdisciplinary and diverse jurisdiction } \\
\text { collaboration }\end{array}$ & $\begin{array}{l}\text { Develop and foster partnership among } \\
\text { internal and external stakeholders. Involve } \\
\text { government agencies in health care, social } \\
\text { services, education, urban development and } \\
\text { housing, private businesses, parents, teachers, } \\
\text { the faith community and the general public. }\end{array}$ \\
\hline $\begin{array}{l}\text { 4. Implement the social and health equity } \\
\text { public policy }\end{array}$ & $\begin{array}{l}\text { Adopt the social and health policy with all } \\
\text { the provisions that address social } \\
\text { determinants of health. }\end{array}$ \\
\hline $\begin{array}{l}\text { 5. Fund innovative research and evaluation } \\
\text { initiatives that analyze the impact of the }\end{array}$ & $\begin{array}{l}\text { Support evidence-based practice through } \\
\text { research funding and advocacy. Scholars, } \\
\text { researchers and policy practitioners evaluate } \\
\text { SHE based on empirical data and best } \\
\text { practices. }\end{array}$ \\
\hline
\end{tabular}

\section{Strategy}

1. Foster a greater indulgent of the social determinants of health among decision makers that progress the adoption of the social and health equity policy.

2. Implement social network and public media strategies to increase awareness among the citizens about health disparities in the United States.

3. Expand programs and support public policies that reduce the social determinants of health that reduce socioeconomic and health inequities in the United States of America.

4. Adopt legislation that enables Americans to access health care through the provision of universal health. Ensure equal opportunities in the allocation of resources and material.

5. Implement cultural competence in the health care and education services by funding and collaborative research initiatives that enhance understanding of social determinants of health. 
6. Institute a multi-stakeholder team to oversee the implementation of recommendations and monitor the impact of adopting the social and health equity policy.

\section{Political Power}

Diligence, expertise and effective advocacy drive the public policy issue of health disparity as defined. Framing the health equity policy as a social justice appeal and lobbing the white house and legislatures will progress bipartisan vote. Intentionality in engaging nongovernmental organizations, interest groups, political parties and the media will create a positive public opinion noted by (Anderson, 2015). Purposeful engagement of the legislatures, committee members, interest groups, and the public will connect the interest of all stakeholders and the benefit-risk variables. Targeted public media address, blogs and forums to create public awareness about the social injustice in health inequality, discrimination, biases and stereotype will stimulate conscious and deliberate actions by the governmental and nongovernmental organizations.

\section{Conclusion}

Sustainable health equity is a promise; a journey that commence with engaging health care providers, clinicians, researches, Government administrators and the citizens in collaborative partnerships for the greater good of individuals and communities. Empirical data and real-life experiences highlight health disparity as a public issue that require innovative approach to succumb the menace. The first step is to mobilize interested parties to build a partnership invested in public health. Demographic, resources, culture, disease prevalence and other relevant data is collected from individuals, and organizations, for equitable health assessment needs, and create a logical model for program a quality healthcare program implementation.

\section{References}

Anderson, J. E. (2015). Public policymaking: An introduction (8th ed.). Stamford, CT: Cengage Learning.

Cook, B., McGuire, T., \&Zaslavsky, A. (2012). Measuring racial/ethnic disparities in health care: methods and practical issues. Health Services Research, 47(3 Pt 2), 1232-1254. https://doi.org/10.1111/j.1475-6773.2012.01387.x

Eltom, S. E., Tchounwou, P., B., \&Rice,V. M. (2011). Bringing new minds and new methods to bridging health disparity and equity. Journal of Healthcare For The Poor And Underserved, 22(4), 3-4. https://doi.org/10.1353/hpu.2011.0167

Esteban, R., \& Dickinson, S. (2013). Obama's health care reform and economic principles of a constitutional issue. RevistaChilena De Economía Y Sociedad, 7(1/2), 40-50.

Feroe, A. G., \&Loebs, S. F. (2015). Reducing Health Disparities through the 2015 MCAT: A Bold Goal Requiring On-going Assessment. Journal Of Health Disparities Research \&Practice, 8(1), 98-105.

Fox Wetle, T., \& Scanlan, K. (2014). Addressing Health Disparities: Brown University School of Public Health. Rhode Island Medical Journal, 97(9), 40-43 


\section{Macrothink}

Journal of Management Research

ISSN 1941-899X 2018, Vol. 10, No. 3

Lundell, H., Niederdeppe, J., \& Clarke, C. (2013). Public Views About Health Causation, Attributions of Responsibility, and Inequality. Journal Of Health Communication, 18(9), 1116-1130. https://doi.org/10.1080/10810730.2013.768724

Miller, H.T. (2011).Is bureaucracy no longer the technically superior form of organization? Administrative Theory and Praxis, 33(3), 447-452. https://doi.org/10.2753/ATP1084-1806330309

Northridge, M. E., \& Freeman, L. (2011). Urban planning and health equity. Journal of Urban Health, 88(3), 582-97. https://doi.org/10.1007/s11524-011-9558-5

Rabiner, M., \& Weiner, A. (2012). Health Care for Homeless and Unstably Housed: Overcoming Barriers. Mount Sinai Journal of Medicine, 79(5), 586-592. https://doi.org/10.1002/msj.21339

Rivera, L. M. (2014). Ethnic-Racial Stigma and Health Disparities: From Psychological Theory and Evidence to Public Policy Solutions. Journal Of Social Issues, 70(2), 198-205. https://doi.org/10.1111/josi.12055

Robelen, E. W. (2011). Frustration at Heart Of Washington Rally. (Cover story). Education Week, 30(35), 1-15.

Siddiqi, A., Hu, X., \& Hall, H. I. (2015). Mortality Among Blacks or African Americans with HIV Infection - United States, 2008-2012. MMWR: Morbidity \& Mortality Weekly Report, 64(4), 81-86.

Srinivasan, S., \& Williams, S. D. (2014). Transitioning from Health Disparities to a Health Equity Research Agenda: The Time Is Now. Public Health Reports, 12971-76. https://doi.org/10.1177/00333549141291S213

Takamura, J. (2014). Closing the Disparity Gap Requires an Integrated Response from Policy, Research, and Programs. Generations, 38(4), 119-126.

Tunkel, A. R. (2014). Health Disparities Education -- The Time Is Now. Rhode Island Medical Journal, 97(9), 21. 